Проблеми математичного моделювання та теорії диференціальних рівнянь

УДК 517.9

\title{
АСИМПТОТИКА РОЗВ'ЯЗКІВ ОДНІЕЇ ЗАДАЧІ ДІРІХЛЕ МЕТОДОМ ПОТЕНЦІАЛІВ
}

\author{
Н. О. Марухно, В. О. Остапенко \\ Дніпропетровсъкий національний університет ім. Олеся Гончара, 49050 , \\ Дніпропетровсък.E-mail: nmaruhno@mail.ru
}

Розглянуто проблему побудови асимптотичного розкладу електричного поля в околі меж. Розв'язання задачі будується у вигляді добутку функцій таким чином, щоб крайові умови на межі області були задовільнені. У випадку нульових крайових умов один зі співмножників у розв'язку обертається на нуль на межі і тому поблизу межі може бути прийнятий як малий параметр. Рівняння Лапласа стає сингуляно-збуреним i це дозволяє будувати розв'язок у вигляді примежового шару. У явному вигляді побудовані асимптотичні розвинення для областей, обмежених колом.

Ключові слова: сингулярно-збурене рівняння, задача Діріхле для кола, асимптотичний розклад розв'язання, потенціал.

\section{1. Вступ}

Будь-яке електричне поле характеризується основними величинами : потенціалом і напругою поля. Напруга електростатичного поля - величина векторна, яка визначається у кожній точці і величиною і напрямком. Потенціал являє собою величину скалярну, значення потенціалу визначається у кожній точці поля деяким числом.

В електростатиці часто виникають задачі знаходження за відомими значеннями потенціалу (або повних зарядів) і геометрії тіл, які утворюють поле, необхідно знайти закон зміни напруги поля і потенціалу в усіх точках поля. У простих випадках задачі на аналітичний розрахунок розв'язують шляхом використання теореми Гаусса в інтегральній формі, у більш складних випадках застосовують рівняння Лапласа. Точний розв'язок такого роду проблеми вдається отримати, головним чином, для окремих випадків, коли геометрія області дозволяє розподілення змінних. Іноді достатньо знати значення електричного поля в деякому околі межі області. Для розв'язання даної задачі застосуємо метод сингулярно-збурених рівнянь, який раніше був застосований для розв'язання задачі про відновлення електромагнітного поля поблизу межі області [3]. У даній статті досліджуються особливості застосування методу сингулярно-збурених рівнянь на прикладі задачі Діріхле для кола і властивості асимптотичних розкладів у примежовому шарі.

(C) Н. О. Марухно, В. О. Остапенко, 2013 


\section{2. Постановка задачі}

Нехай двовимірна область $G$ має межу $S$, що складається з частин різних кривих, тобто $S=\bigcup_{j=1}^{n} S_{j}$. В цій області необхідно знайти двічі неперервнодиференційований розв'язок рівняння Лапласа:

$$
\Delta u=0,
$$

що задовольняє крайовій умові:

$$
\left.u(x, y)\right|_{S}=g(x, y) .
$$

\section{3. Розв'язання задачі}

Розв'язання задачі (2.1)-(2.2) будується на основі можливості подання двічі неперервно-диференційованої функції у внутрішніх точках області у вигляді суми потенціалів [4]:

$$
u\left(z_{0}\right)=\int_{S}\left(-\delta\left(z, z_{0}\right) \frac{\partial u(z)}{\partial n}+u \frac{\partial \delta\left(z, z_{0}\right)}{\partial n}\right) d S+\iint_{G} \delta\left(z, z_{0}\right) \Delta u d G .
$$

Для застосування формули (3.1) до розв'язання поставленої задачі не вистачає одного доданка:

$$
\left.\frac{\partial u}{\partial n}\right|_{S}=\Theta(z) .
$$

На основі умов постановки задачі обчислити значення похідної шуканої функції за напрямком зовнішньої нормалі неможливо, оскільки зміни шуканої функції уздовж зовнішньої нормалі не задані. Тому ставиться допоміжна задача визначити значення функції $u(x, y)$ в околі межі області, за допомогою асимптотичного подання значення функції $u(x, y)$.

Якщо кожна $j$-та частина границі $S$ являе собою криву, задану у неявному вигляді $f_{j}(x, y)=0, j=1,2, \ldots, n$, то в околі частини такої кривої розв'язок шукається у такому вигляді:

$$
u(x, y)=\gamma_{i}\left(\alpha_{i} f_{i}\right) v(x, y)+k_{j}\left(\beta_{j} f_{j}\right) g(x, y),
$$

де $k_{j}, \gamma_{j}$ - довільні функції, що задовольняють умовам $k_{j}(0)=1, \gamma_{j}(0)=$ $0, \alpha_{i}, \beta_{j}$ - довільні числа.

Підставляючи форму розв'язку (3.2) у рівняння (2.1), одержимо рівність:

$$
\begin{gathered}
\gamma_{j}\left(\frac{\partial^{2} v}{\partial x^{2}}+\frac{\partial^{2} v}{\partial y^{2}}\right)+2\left(\frac{\partial \gamma_{j}}{\partial x} \frac{\partial v}{\partial x}+\frac{\partial \gamma_{j}}{\partial y} \frac{\partial v}{\partial y}\right)+\left(\frac{\partial^{2} \gamma_{j}}{\partial x^{2}}+\frac{\partial^{2} \gamma_{j}}{\partial y^{2}}\right) v+ \\
\left(\frac{\partial^{2} k_{j}}{\partial x^{2}}+\frac{\partial^{2} k_{j}}{\partial y^{2}}\right) g+k_{j}\left(\frac{\partial^{2} g}{\partial x^{2}}+\frac{\partial^{2} g}{\partial y^{2}}\right)+2\left(\frac{\partial k_{j}}{\partial x} \frac{\partial g}{\partial x}+\frac{\partial k_{j}}{\partial y} \frac{\partial g}{\partial y}\right)=0 .
\end{gathered}
$$


У безпосередній близькості до частини границі $S_{j}$ функція $\gamma_{j}$ може бути скільки завгодно близькою до нуля, тому в такому околі її можна розглядати як малий параметр. У цьому випадку рівняння (3.3) стає сингулярнозбуреним. Це дозволяє, поклавши $\gamma_{j}=\epsilon$, подати рівняння (3.3) у вигляді:

$$
\begin{gathered}
2\left(\frac{\partial \gamma_{j}}{\partial x} \frac{\partial v}{\partial x}+\frac{\partial \gamma_{j}}{\partial y} \frac{\partial v}{\partial y}\right)+\left(\frac{\partial^{2} \gamma_{j}}{\partial x^{2}}+\frac{\partial^{2} \gamma_{j}}{\partial y^{2}}\right) v+\left(\frac{\partial^{2} k_{j}}{\partial x^{2}}+\frac{\partial^{2} k_{j}}{\partial y^{2}}\right) g+ \\
k_{j}\left(\frac{\partial^{2} g}{\partial x^{2}}+\frac{\partial^{2} g}{\partial y^{2}}\right)+2\left(\frac{\partial k_{j}}{\partial x} \frac{\partial g}{\partial x}+\frac{\partial k_{j}}{\partial y} \frac{\partial g}{\partial y}\right)=-\epsilon\left(\frac{\partial^{2} v}{\partial x^{2}}+\frac{\partial^{2} v}{\partial y^{2}}\right) .
\end{gathered}
$$

У примежовому шарі будується асимптотичний розклад розв'язку рівняння (3.4):

$$
v(x, y)=\sum_{i=0}^{\infty} \epsilon^{i} v_{i}(x, y) .
$$

Підстановка форми розв'язку (3.5) у рівняння (3.4) і прирівнювання коефіцієнтів при відповідних степенях $\epsilon$ приводить до системи диференційних рівнянь першого порядку з частинними похідними:

$$
\begin{gathered}
2\left(\frac{\partial \gamma_{j}}{\partial x} \frac{\partial v_{0}}{\partial x}+\frac{\partial \gamma_{j}}{\partial y} \frac{\partial v_{0}}{\partial y}\right)+\left(\frac{\partial^{2} \gamma_{j}}{\partial x^{2}}+\frac{\partial^{2} \gamma_{j}}{\partial y^{2}}\right) v_{0}+\left(\frac{\partial^{2} k_{j}}{\partial x^{2}}+\frac{\partial^{2} k_{j}}{\partial y^{2}}\right) g+ \\
k_{j}\left(\frac{\partial^{2} g}{\partial x^{2}}+\frac{\partial^{2} g}{\partial y^{2}}\right)+2\left(\frac{\partial k_{j}}{\partial x} \frac{\partial g}{\partial x}+\frac{\partial k_{j}}{\partial y} \frac{\partial g}{\partial y}\right)=0 \\
2\left(\frac{\partial \gamma_{j}}{\partial x} \frac{\partial v_{i}}{\partial x}+\frac{\partial \gamma_{j}}{\partial y} \frac{\partial v_{i}}{\partial y}\right)+\left(\frac{\partial^{2} \gamma_{j}}{\partial x^{2}}+\frac{\partial^{2} \gamma_{j}}{\partial y^{2}}\right) v_{i}=-\left(\frac{\partial^{2} v_{i-1}}{\partial x^{2}}+\frac{\partial^{2} v_{i-1}}{\partial y^{2}}\right) .
\end{gathered}
$$

Рівняння (3.6) є породжувальним. Для знаходження функції $v_{0}$ необхідно вибрати функцію $\gamma_{j}$, що задовольняє перерахованим вище умовам. У задачах електромагнетизму краще за всіх підходить функція $\sin x$. Як функцію $k_{j}$ можна вибрати $\cos x$, вона також задовольняе вищезазначеним умовам.

Рівняння (3.6 )перетворенням:

$$
\frac{\partial v_{0}}{\partial x}=-\frac{\frac{\partial w_{0}}{\partial x}}{\frac{\partial w_{0}}{\partial v_{0}}} ; \frac{\partial v_{0}}{\partial y}=-\frac{\frac{\partial w_{0}}{\partial y}}{\frac{\partial w_{0}}{\partial v_{0}}},
$$

де $w_{0}\left(v_{0}(x, y), x, y\right)=$ const - перший інтеграл рівняння(3.6), приводиться до рівняння:

$$
\begin{gathered}
2\left(\frac{\partial \gamma_{j}}{\partial x} \frac{\partial w_{0}}{\partial x}+\frac{\partial \gamma_{j}}{\partial y} \frac{\partial w_{0}}{\partial y}\right)-\left(\frac{\partial^{2} \gamma_{j}}{\partial x^{2}}+\frac{\partial^{2} \gamma_{j}}{\partial y^{2}}\right) v_{0}+\left(\frac{\partial^{2} k_{j}}{\partial x^{2}}+\frac{\partial^{2} k_{j}}{\partial y^{2}}\right) g+ \\
k_{j}\left(\frac{\partial^{2} g}{\partial x^{2}}+\frac{\partial^{2} g}{\partial y^{2}}\right)+2\left(\frac{\partial k_{j}}{\partial x} \frac{\partial g}{\partial x}+\frac{\partial k_{j}}{\partial y} \frac{\partial g}{\partial y}\right) \frac{\partial w_{0}}{\partial v_{0}}=0
\end{gathered}
$$


якому відповідає система звичайних диференціальних рівнянь

$$
\begin{gathered}
\frac{d x}{2 \frac{\partial \gamma_{j}}{\partial x}}=\frac{d y}{2 \frac{\partial \gamma_{j}}{\partial y}}= \\
=\frac{-d v_{0}}{\left(\frac{\partial^{2} \gamma_{j}}{\partial x^{2}}+\frac{\partial^{2} \gamma_{j}}{\partial y^{2}}\right) v_{0}+\left(\frac{\partial^{2} k_{j}}{\partial x^{2}}+\frac{\partial^{2} k_{j}}{\partial y^{2}}\right) g+k_{j}\left(\frac{\partial^{2} g}{\partial x^{2}}+\frac{\partial^{2} g}{\partial y^{2}}\right)+2\left(\frac{\partial k_{j}}{\partial x} \frac{\partial g}{\partial x}+\frac{\partial k_{j}}{\partial y} \frac{\partial g}{\partial y}\right)} .
\end{gathered}
$$

Ліва пара рівняння (3.10) має перший інтеграл:

$$
\Psi(x)=C .
$$

Якщо (3.11) можна розв'язати відносно $y$ (або $x$ ), то одержимо:

$$
y=f(x, y),
$$

пара крайніх рівнянь (3.10) приводиться до лінійного диференціального рівняння:

$$
\begin{gathered}
\frac{d v_{0}}{d x}+\frac{\left(\frac{\partial^{2} \gamma_{j}}{\partial x^{2}}+\frac{\partial^{2} \gamma_{j}}{\partial y^{2}}\right) v_{0}}{2 \frac{\partial \gamma_{j}}{\partial x}}= \\
=-\frac{\left(\frac{\partial^{2} k_{j}}{\partial x^{2}}+\frac{\partial^{2} k_{j}}{\partial y^{2}}\right) g+k_{j}\left(\frac{\partial^{2} g}{\partial x^{2}}+\frac{\partial^{2} g}{\partial y^{2}}\right)+2\left(\frac{\partial k_{j}}{\partial x} \frac{\partial g}{\partial x}+\frac{\partial k_{j}}{\partial y} \frac{\partial g}{\partial y}\right)}{2 \frac{\partial \gamma_{j}}{\partial x}} .
\end{gathered}
$$

Нехай виконується рівність (3.12). Рівняння (3.13) - лінійне неоднорідне, тому його загальний розв'язок з використанням рівності (3.12) має вигляд:

$$
\begin{gathered}
v_{0}(x, y)=\exp \left(-\int \frac{\frac{\partial^{2} \gamma_{j}}{\partial x^{2}}+\frac{\partial^{2} \gamma_{j}}{\partial y^{2}}}{2 \frac{\partial \gamma_{j}}{\partial x}} d x\right)\left[C_{0}-\right. \\
-\int \frac{\left(\frac{\partial^{2} k_{j}}{\partial x^{2}}+\frac{\partial^{2} k_{j}}{\partial y^{2}}\right) g+k_{j}\left(\frac{\partial^{2} g}{\partial x^{2}}+\frac{\partial^{2} g}{\partial y^{2}}\right)+2\left(\frac{\partial k_{j}}{\partial x} \frac{\partial g}{\partial x}+\frac{\partial k_{j}}{\partial y} \frac{\partial g}{\partial y}\right)}{2 \frac{\partial \gamma_{j}}{\partial x}} \\
\left.\exp \left(\int \frac{\frac{\partial^{2} \gamma_{j}}{\partial x^{2}}+\frac{\partial^{2} \gamma_{j}}{\partial y^{2}}}{2 \frac{\partial \gamma_{j}}{\partial x}} d x\right) d x\right] .
\end{gathered}
$$

Рівняння (3.7) перетворенням:

$$
\frac{\partial v_{i}}{\partial x}=-\frac{\frac{\partial w_{i}}{\partial x}}{\frac{\partial w_{i}}{\partial v_{i}}} ; \frac{\partial v_{i}}{\partial y}=-\frac{\frac{\partial w_{i}}{\partial y}}{\frac{\partial w_{i}}{\partial v_{i}}},
$$

де $w_{i}\left(v_{i}(x, y), x, y\right)=$ const - перший інтеграл рівняння $(3.7)$, приводиться до рівняння: 
$2\left(\frac{\partial \gamma_{j}}{\partial x} \frac{\partial w_{i}}{\partial x}+\frac{\partial \gamma_{j}}{\partial y} \frac{\partial w_{i}}{\partial y}\right)-\left[\left(\frac{\partial^{2} \gamma_{j}}{\partial x^{2}}+\frac{\partial^{2} \gamma_{j}}{\partial y^{2}}\right) v_{i}+\frac{\partial^{2} v_{i-1}}{\partial x^{2}}+\frac{\partial^{2} v_{i-1}}{\partial y^{2}}\right] \frac{\partial w_{i}}{\partial v_{i}}$

якому відповідає система звичайних диференціальних рівнянь:

$$
\frac{d x}{2 \frac{\partial \gamma_{j}}{\partial x}}=\frac{d y}{2 \frac{\partial \gamma_{j}}{\partial y}}=\frac{-d v_{i}}{\left(\frac{\partial^{2} \gamma_{j}}{\partial x^{2}}+\frac{\partial^{2} \gamma_{j}}{\partial y^{2}}\right) v_{i}+\frac{\partial^{2} v_{i-1}}{\partial x^{2}}+\frac{\partial^{2} v_{i-1}}{\partial y^{2}}} .
$$

Ліва пара рівняння (3.17) має перший інтеграл:

$$
\Phi(x, y)=C .
$$

Якщо (3.18) можна розв'язати відносно $y($ або $x)$, то одержимо:

$$
y=f(x) .
$$

Пара крайніх рівнянь (3.17) приводиться до лінійних диференціальних рівнянь:

$$
\frac{d v_{i}}{d x}+\frac{\left(\frac{\partial^{2} \gamma_{j}}{\partial x^{2}}+\frac{\partial^{2} \gamma_{j}}{\partial y^{2}}\right) v_{i}}{2 \frac{\partial \gamma_{j}}{\partial x}}=-\frac{\frac{\partial^{2} v_{i-1}}{\partial x^{2}}+\frac{\partial^{2} v_{i-1}}{\partial y^{2}}}{\frac{\partial \gamma_{j}}{\partial x}}, i=1,2, \ldots
$$

Нехай виконується рівність (3.19), аналогічно до рівняння (3.10) розв'язуємо рівняння (3.20). Загальний розв'язок кожного з рівнянь (3.20) при використанні рівності(3.18) має вигляд:

$$
\begin{gathered}
v_{i}(x, y)=\exp \left(-\int \frac{\frac{\partial^{2} \gamma_{j}}{\partial x^{2}}+\frac{\partial^{2} \gamma_{j}}{\partial y^{2}}}{2 \frac{\partial \gamma_{j}}{\partial x}} d x\right)\left[C_{i}-\right. \\
\left.-\int \frac{\frac{\partial^{2} v_{i-1}}{\partial x^{2}}+\frac{\partial^{2} v_{i-1}}{\partial y^{2}}}{\frac{\partial \gamma_{j}}{\partial x}} \exp \left(\int \frac{\frac{\partial^{2} \gamma_{j}}{\partial x^{2}}+\frac{\partial^{2} \gamma_{j}}{\partial y^{2}}}{2 \frac{\partial \gamma_{j}}{\partial x}} d x\right) d x\right] .
\end{gathered}
$$

Підставивши вирази для $v_{i}(x, y)$ в $(3.5)$, ми одержимо асимптотичний розклад функції $v(x, y)$.

\section{4. Застосування методу для області, обмеженної колом}

У ході практичного застосування методу сингулярно-збурених рівнянь у декартових координатах для області, обмеженної колом, було знайдено вираз для функції $v_{0}$, він дуже громіздкий, містить гіпергеометричні функції та еліптичний інтеграл другого роду, і у подальшому ці функції необхідно буде ще й інтегрувати і диференціювати, тому доцільно буде перейти до полярної системи координат, що значно полегшить роботу і надасть більш комфортний для застосування вираз шуканої функції $u(z)$. 
Запишемо рівняння Лапласа (2.1) у полярній системі координат.

$$
\Delta u=\frac{1}{\rho} \frac{\partial u}{\partial \rho}+\frac{\partial^{2} u}{\partial^{2} \rho}+\frac{1}{\rho^{2}} \frac{\partial^{2} u}{\partial \varphi^{2}}=0,
$$

а функції $k, \gamma, f$ набувають вигляду:

$$
\gamma=\sin (R-\rho), k=\cos (R-\rho), f=R-\rho .
$$

Оскільки функцію шукаємо у вигляді:

$$
u(z)=\gamma(f) v(z)+k(f) g(z),
$$

то, диференціюючи їі, одержимо:

$$
\begin{gathered}
\frac{\partial u}{\partial \rho}=-\cos (R-\rho) v(\rho, \varphi)+\sin (R-\rho) \frac{\partial v}{\partial \rho}+\sin (R-\rho) g(R, \varphi) \\
\frac{\partial^{2} u}{\partial \rho^{2}}=-\sin (R-\rho) v(\rho, \varphi)-2 \cos (R-\rho) \frac{\partial v}{\partial \rho}-\cos (R-\rho) g(R, \varphi)+ \\
+\sin (R-\rho) \frac{\partial^{2} v}{\partial \rho^{2}} \\
\frac{\partial^{2} u}{\partial \varphi^{2}}=\sin (R-\rho) \frac{\partial^{2} v}{\partial \varphi^{2}}+\cos (R-\rho) \frac{\partial^{2} g}{\partial \varphi^{2}}
\end{gathered}
$$

При розрахунку похідних враховано, що на межі функція $g(z)$ від $R$ не залежить, тобто $g(z)=g(\varphi)$. Підставивши ці похідні у рівняння (4.1), одержимо:

$$
\begin{gathered}
\cos (R-\rho)\left[-\frac{v}{\rho}-2 \frac{\partial v}{\partial \rho}-g(R, \varphi)+\frac{1}{\rho^{2}} \frac{\partial^{2} g}{\partial \varphi^{2}}\right]= \\
-\sin (R-\rho)\left[\frac{1}{\rho} \frac{\partial v}{\partial \rho}-v+\frac{g(R, \varphi)}{\rho}+\frac{\partial^{2} v}{\partial \rho^{2}}+\frac{1}{\rho^{2}} \frac{\partial^{2} v}{\partial \rho^{2}}\right]
\end{gathered}
$$

або

$$
\begin{gathered}
\frac{v}{\rho}+2 \frac{\partial v}{\partial \rho}+g(R, \varphi)-\frac{1}{\rho^{2}} \frac{\partial^{2} g}{\partial \varphi^{2}}= \\
\tan (R-\rho)\left[\frac{1}{\rho} \frac{\partial v}{\partial \rho}-v+\frac{g(R, \varphi)}{\rho}+\frac{\partial^{2} v}{\partial \rho^{2}}+\frac{1}{\rho^{2}} \frac{\partial^{2} v}{\partial \rho^{2}}\right] .
\end{gathered}
$$

У безпосередній близькості до межі $\tan (R-\rho)$ може бути скільки завгодно малим, тому в такому околі його можна розглядати як малий параметр.

У цьому випадку рівняння (4.1) стає сингулярно-збуреним, це можливо, позначивши:

$$
\tan (R-\rho)=\epsilon .
$$

Одержимо наступне рівняння:

$$
\frac{v}{\rho}+2 \frac{\partial v}{\partial \rho}+g(R, \varphi)-\frac{1}{\rho^{2}} \frac{\partial^{2} g}{\partial \varphi^{2}}=
$$




$$
=\epsilon\left[\frac{1}{\rho} \frac{\partial v}{\partial \rho}-v+\frac{g(R, \varphi)}{\rho}+\frac{\partial^{2} v}{\partial \rho^{2}}+\frac{1}{\rho^{2}} \frac{\partial^{2} v}{\partial \rho^{2}}\right] .
$$

У примежовому шарі будується асимптотичний розклад розв'язку рівняння:

$$
v(z)=\sum_{i=0}^{\infty} \epsilon^{i} v_{i}(z) .
$$

Підстановка форми розв'язку (4.7) у рівняння (4.6) і прирівнювання у правій та лівій частинах одержаного рівняння коефіцієнтів при однакових степенях $\epsilon$ приводить до системи диференційних рівнянь першого порядку 3 частинними похідними:

$$
\begin{gathered}
\epsilon^{0}: \frac{v_{0}}{\rho}+2 \frac{\partial v_{0}}{\partial \rho}=-g(R, \varphi)+\frac{1}{\rho^{2}} \frac{\partial^{2} g}{\partial \varphi^{2}} \\
\epsilon^{1}: \frac{v_{1}}{\rho}+2 \frac{\partial v_{1}}{\partial \rho}=\frac{\partial^{2} v_{0}}{\partial \rho^{2}}+\frac{1}{\rho} \frac{\partial v_{0}}{\partial \rho}-v_{0}+\frac{g(R, \varphi)}{\rho}+\frac{1}{\rho^{2}} \frac{\partial^{2} v_{0}}{\partial \varphi^{2}} \\
\epsilon^{2}: \frac{v_{2}}{\rho}+2 \frac{\partial v_{2}}{\partial \rho}=\frac{\partial^{2} v_{1}}{\partial \rho^{2}}+\frac{1}{\rho} \frac{\partial v_{1}}{\partial \rho}-v_{1}+\frac{1}{\rho^{2}} \frac{\partial^{2} v_{1}}{\partial \varphi^{2}} .
\end{gathered}
$$

Продовжуючи аналогічно до попередніх виразів, одержимо загальну формулу для $i=2,3, \ldots$ :

$$
\epsilon^{i}: \frac{v_{i}}{\rho}+2 \frac{\partial v_{i}}{\partial \rho}=\frac{\partial^{2} v_{i-1}}{\partial \rho^{2}}+\frac{1}{\rho} \frac{\partial v_{i-1}}{\partial \rho}-v_{i-1}+\frac{1}{\rho^{2}} \frac{\partial^{2} v_{i-1}}{\partial \varphi^{2}} .
$$

Розв'язуючи перше рівняння системи аналогічно формулі для декартових прямокутних координат, запишемо вираз для $v_{i}$ у полярній системі:

$$
\begin{gathered}
v_{0}(z)=\exp \left(-\int \frac{d \rho}{2 \rho}\right)\left[C_{0}(\varphi)+\int\left(\left[-g(R, \varphi)+\frac{1}{\rho^{2}}+\frac{\partial^{2} g}{\varphi^{2}}\right] \exp \left(\int \frac{d \rho}{2 \rho}\right)\right) d \rho\right] \\
v_{1}(z)=\exp \left(-\int \frac{d \rho}{2 \rho}\right)\left[C_{1}(\varphi)+\int\left[\left[\frac{\partial^{2} v_{0}}{\partial \rho^{2}}+\frac{1}{\rho} \frac{\partial v_{0}}{\partial \rho}-v_{0}+\frac{g(R, \varphi)}{\rho}+\frac{1}{\rho^{2}} \frac{\partial^{2} v_{0}}{\partial \varphi^{2}}\right]\right.\right. \\
\left.\left.\exp \left(\int \frac{d \rho}{2 \rho}\right)\right] d \rho\right] ; \\
v_{i}(z)=\exp \left(-\int \frac{d \rho}{2 \rho}\right)\left[C_{i}(\varphi)+\int\left[\left[\frac{\partial^{2} v_{i-1}}{\partial \rho^{2}}+\frac{1}{\rho} \frac{\partial v_{i-1}}{\partial \rho}-v_{i-1}+\frac{1}{\rho^{2}} \frac{\partial^{2} v_{i-1}}{\partial \varphi^{2}}\right]\right.\right. \\
\left.\left.\exp \left(\int \frac{d \rho}{2 \rho}\right)\right] d \rho\right]
\end{gathered}
$$

Враховуючи, що

$$
\exp \left(\int \frac{d \rho}{2 \rho}\right)=\exp \left(\frac{1}{2} \ln \rho\right)=\sqrt{\rho},
$$


одержимо з (4.10):

$$
\begin{gathered}
v_{0}(z)=\frac{1}{\sqrt{\rho}}\left[C_{0}(\varphi)+\int\left(\left[-g(R, \varphi)+\frac{1}{\rho^{2}}+\frac{\partial^{2} g}{\varphi^{2}}\right] \sqrt{\rho}\right) d \rho\right], \\
v_{1}(z)=\frac{1}{\sqrt{\rho}}\left[C_{1}(\varphi)+\int\left(\left[\frac{\partial^{2} v_{0}}{\partial \rho^{2}}+\frac{1}{\rho} \frac{\partial v_{0}}{\partial \rho}-v_{0}+\frac{g(R, \varphi)}{\rho}+\frac{1}{\rho^{2}} \frac{\partial^{2} v_{0}}{\partial \varphi^{2}}\right] \sqrt{\rho}\right) d \rho\right], \\
v_{i}(z)=\frac{1}{\sqrt{\rho}}\left[C_{i}(\varphi)+\int\left[\left[\frac{\partial^{2} v_{i-1}}{\partial \rho^{2}}+\frac{1}{\rho} \frac{\partial v_{i-1}}{\partial \rho}-v_{i-1}+\frac{1}{\rho^{2}} \frac{\partial^{2} v_{i-1}}{\partial \varphi^{2}}\right] \sqrt{\rho}\right] d \rho\right]
\end{gathered}
$$

Враховуючи, що:

$$
\int \sqrt{\rho} d \rho=\frac{2}{3} \rho^{\frac{3}{2}}, \quad \int \rho^{\frac{-3}{2}} d \rho=\frac{-2}{\sqrt{\rho}},
$$

одержимо з (4.11):

$$
v_{0}(z)=\frac{C_{0}(\varphi)}{\sqrt{\rho}}-\frac{g(R, \varphi)}{3}+\frac{1}{\rho^{2}} \frac{\partial^{2} g}{\partial \varphi^{2}}
$$

Із (4.12) випливає, що:

$$
\frac{\partial v_{0}(z)}{\partial \rho}=-\frac{C_{0}(\varphi)}{2} \rho^{\frac{-3}{2}}-\frac{g(R, \varphi)}{3}+\frac{1}{\rho^{2}} \frac{\partial^{2} g}{\partial \varphi^{2}}
$$

Тому з (4.13) і виразів для похідних одержимо:

$$
\begin{gathered}
\frac{\partial u_{0}(z)}{\partial \rho}=-\cos (R-\rho)\left[\frac{C_{0}(\varphi)}{\sqrt{\rho}}-\frac{g(R, \varphi)}{3}+\frac{1}{\rho^{2}} \frac{\partial^{2} g}{\partial \varphi^{2}}\right]+ \\
+\sin (R-\rho)\left[-\frac{C_{0}(\varphi)}{2} \rho^{\frac{-3}{2}}-\frac{g(R, \varphi)}{3}+\frac{1}{\rho^{2}} \frac{\partial^{2} g}{\partial \varphi^{2}}\right]+\sin (R-\rho) g(R, \varphi) .
\end{gathered}
$$

Із (4.14) випливає, що:

$$
\left.\frac{\partial u_{0}(z)}{\partial \rho}\right|_{S}=-\left[\frac{C_{0}(\varphi)}{\sqrt{\rho}}-\frac{g(R, \varphi)}{3}+\frac{1}{\rho^{2}} \frac{\partial^{2} g}{\partial \varphi^{2}}\right]
$$

На площині функція одиничного джерела визначається таким чином:

$$
\delta\left(z, z_{0}\right)=\frac{1}{2 \pi} \ln \sqrt{\rho^{2}+r_{0}^{2}-2 r_{0} \rho \cos (\beta)},
$$

$\beta$ - кут між напрямками $r_{0}$ і $\rho$. Тому

$$
\left.\frac{\partial \delta\left(z, z_{0}\right)}{\partial \rho}\right|_{S}=\frac{1}{2 \pi} \frac{R-r_{0} \cos (\beta)}{R^{2}+\rho_{0}^{2}-2 \rho r_{0} \cos (\beta)} .
$$


Отже, відповідно до формули (3.1) одержимо нульове наближення розв'язку задачі:

$$
\begin{gathered}
u\left(p_{0}\right)=\int_{0}^{2 \pi} \frac{R}{2 \pi} \ln \sqrt{R^{2}+r_{0}^{2}-2 R r_{0} \cos (\beta)}\left[\frac{C_{0}(\varphi)}{\sqrt{R}}-\frac{g(R, \varphi)}{3} R-\frac{1}{R} \frac{\partial^{2} g}{\partial \varphi^{2}}\right] d \varphi+ \\
+\int_{0}^{2 \pi} \frac{g(R, \varphi)}{2 \pi} * \frac{R\left(R-r_{0} \cos (\beta)\right)}{R^{2}+r_{0}^{2}-2 R r_{0} \cos (\beta)} d \varphi .
\end{gathered}
$$

При обчисленні інтегралів типу (4.15) необхідно враховувати, що кут $\beta$ як функція від кута $\varphi$ залежить від координат точки $z_{0}:\left(x_{0}, y_{0}\right)$, а саме $\beta=$ $\varphi-\alpha$, де

$$
\alpha=\left\{\begin{array}{l}
\operatorname{arctg} \frac{y_{0}-b}{x_{0}-a}, x_{0}-a \geq 0, y_{0}-b \geq 0, \\
\pi-\operatorname{arctg} \frac{y_{0}-b}{-\left(x_{0}-a\right)}=\frac{\pi}{2}+\operatorname{arctg} \frac{-\left(x_{0}-a\right)}{y_{0}-b}, x_{0}-a \leq 0, y_{0}-b \geq 0, \\
\pi+\operatorname{arctg} \frac{y_{0}-b}{x_{0}-a}=\frac{3 \pi}{2}-\operatorname{arctg} \frac{x_{0}-a}{y_{0}-b}, x_{0}-a \leq 0, y_{0}-b \leq 0, \\
2 \pi-\operatorname{arctg} \frac{-\left(y_{0}-b\right)}{x_{0}-a}=\frac{3 \pi}{2}+\operatorname{arctg} \frac{x_{0}-a}{-\left(y_{0}-b\right)}, x_{0}-a \geq 0, y_{0}-b \leq 0 .
\end{array}\right.
$$

Тут $(a, b)$ - координати центра кола.

Функції $C_{i}(\varphi)$ визначаються із властивості гармонічної функції $u(z)$ :

$$
\int_{S} \frac{\partial u(z)}{\partial n} d S=0
$$

Враховуючи подання функції $u(z)$ (4.3) і асимптотичний розклад $v(z)$ (4.7), функцію $u(z)$ можна подати у вигляді асимптотичного розкладу

$$
u(z)=u_{0}(z)+u_{1}(z) \epsilon+u_{2} \epsilon^{2}+\ldots,
$$

де

$$
u_{0}(z)=\gamma(f) v_{0}+k(f) g(z) ; \quad u_{i}=\gamma(f) v_{i}(z), i=0,1, \ldots
$$

Із підстановки функції (4.3) у (4.4) випливає:

$$
\int_{S} \frac{\partial u_{i}(z)}{\partial n} d S=0, i=0,1, \ldots
$$

Рівності (4.19) слугують умовами для знаходження функцій $C_{i}(\varphi)$.

Запишемо вираз для $C_{0}(\varphi)$ відповідно до формули (4.19).

Підставимо вираз для похідної, одержимо наступне інтегральне рівняння для знаходження $C_{0}(\varphi)$ :

$$
\int_{0}^{2 \pi}\left[-\sqrt{R} C_{0}(\varphi)+\frac{R g(R, \varphi)}{3} R+\frac{\partial^{2} g}{\partial \varphi^{2}}\right] d \varphi=0 .
$$


Аналогічно записуються інтегральні рівняння для знаходження $C_{i}(\varphi)$.

\section{5. Застосування методу для чисельних прикладів}

Розглянемо наступну крайову задачу. У колі радіуса $R$ із центром у початку координат необхідно знайти двічі неперервно-диференційований розв'язок рівняння Лапласа:

$$
\Delta u(x, y)=0
$$

що задовольняе умові

$$
\left.u(x, y)\right|_{S}=R(\cos (\varphi)+\sin (\varphi)) .
$$

Застосуємо формулу для першого наближення (4.15) для конкретних точок $p_{0}$ і порівняємо з точним розв'язком задачі Діріхле для кола:

$$
u\left(z_{0}\right)=\frac{1}{2 \pi} \int_{0}^{2 \pi} \Phi\left(R e^{i \varphi}\right) d \varphi=\frac{R^{2}+r_{0}^{2}}{R^{2}+r_{0}^{2}-2 R r_{0} \cos \left(\varphi-\varphi_{0}\right)} d \varphi
$$

де $r_{0}=\left|z_{0}\right|, \varphi_{0}=\arg z_{0}$.

Відповідне значення для $C_{0}(\varphi)$ знайдемо з інтегрального рівняння (4.20), враховуючи значення функції $g(R, \varphi)$ :

$$
\int_{0}^{2 \pi}\left[-\sqrt{R} C_{0}(\varphi)+\frac{R^{2}(\cos (\varphi)+\sin (\varphi))}{3} R+\frac{R \partial^{2}(\cos (\varphi)+\sin (\varphi))}{\partial^{2} \varphi}\right] d \varphi=0 .
$$

Рівняння (5.3) має безліч розв'язків. 3 одного боку, це дає нам можливість підібрати вираз для $C_{0}(\varphi)$ таким чином, щоб значення першого наближення якнайкраще наближувалось до точного значення функцій у вибраній точці.

3 іншого боку, це не $є$ добре, оскільки вся точність даного методу залежатиме саме від вибору $C_{i}(\varphi)$.

Для нашої задачі перше наближення запишеться за такою формулою:

$$
\begin{gathered}
u\left(p_{0}\right)=\int_{0}^{2 \pi} \frac{\ln \left(R^{2}+r_{0}^{2}-2 R r_{0} \cos \beta\right)}{2 \pi}\left[\frac{C_{0}}{\sqrt{R}}+\left(-\frac{R^{2}}{3}+1\right)(\cos \varphi+\sin \varphi)\right] d \varphi+ \\
+\int_{0}^{2 \pi} \frac{(\cos (\varphi)+\sin (\varphi))}{2 \pi} \frac{\left(R^{2}-2 R r_{0} \cos (\beta)\right)}{R^{2}+r_{0}^{2}-2 R r_{0} \cos (\beta)} d \varphi .
\end{gathered}
$$

Розв'язуючи перший інтеграл виразу (5.4) по частинах, одержимо такий вираз:

$$
\begin{gathered}
\int_{0}^{2 \pi} \frac{1}{2 \pi}\left(-\frac{R^{2}}{3}+1\right) \ln \left(R^{2}+r_{0}^{2}-2 R r_{0} \cos (\varphi)\right)(\cos (\varphi)+\sin (\varphi)) d \varphi= \\
=-\int_{0}^{2 \pi} \frac{1}{2 \pi}\left(-\frac{R^{2}}{3}+1\right) \frac{2 R r_{0} \sin ^{2}(\varphi)}{R^{2}+r_{0}^{2}-2 R r_{0} \cos (\beta)} d \varphi+
\end{gathered}
$$




$$
+\int_{0}^{2 \pi} \frac{1}{2 \pi}\left(-\frac{R^{2}}{3}+1\right) \frac{2 R r_{0} \sin (\varphi) \cos (\varphi)}{R^{2}+r_{0}^{2}-2 R r_{0} \cos (\beta)} d \varphi .
$$

Під час підрахунків значень першого наближення було помічено, що другий інтеграл виразу (5.4) дорівнюе половині значення точного розв'язку, тобто перший інтеграл також повинен дорівнювати половині точного значення розв'язку. Залучаючи даний висновок за $C_{0}(\varphi)$, виберемо $\cos (\varphi)$, оскільки саме такий вираз для константи найкраще наближує значення першого інтеграла до половини точного значення розв'язку. Для підтвердження доцільності застосування методу сингулярно-збурених диференційних рівнянь для розв'язку задачі Діріхле для рівняння Лапласа для кола розглянемо наступні чисельні приклади.

Розрахунки проводимо за допомогою програми MATLAB.

1. Вихідні дані: $R=2, r=1.1000, T=0$.

Асимптотичний розв'язок у точці $p_{0}: u_{0}=1.1222$.

Точний розв' язок у точці $p_{0}: u_{0}=1.1000$.

Абсолютна похибка: $c=0.0222$. Відносна похибка: $c=0.02018$.

2. Вихідні дані: $R=2, r=1.1000, T=1.5708$.

Асимптотичний розв'язок у точці $p_{0}: u_{0}=0.7333$.

Точний розв'язок у точці $p_{0}: u_{0}=1.1000$.

Абсолютна похибка: $c=0.3667$. Відносна похибка $c=0.33$.

3. Вихідні дані: $R=2, r=1.4000, T=\pi$.

Асимптотичний розв'язок у точці $p_{0}: u_{0}=-1.4283$.

Точний розв' язок у точці $p_{0}: u_{0}=-1.4000$.

Абсолютна похибка: $c=0.0283$. Відносна похибка: $c=0.0202$.

4. Вихідні дані: $R=2, r=1.6000, T=13.3518$.

Асимптотичний розв'язок у точці $p_{0}: u_{0}=1.9085$.

Точний розв' язок у точці $p_{0}: u_{0}==2.2627$.

Абсолютна похибка: $c=0.3542$. Відносна похибка: $c=0.1565$.

Одержані результати показують, що перше наближення шуканої функції дуже близьке до точного розв'язку крайової задачі. Точного збігу можна досягти за рахунок малого параметра $\epsilon$ та наступних наближень шуканої функції.

\section{6. Висновки}

Метод сингулярно-збурених диференціальних рівнянь дозволяе будувати асимптотичні розклади розв'язків крайових задач у примежовому шарі зі 
скільки завгодно великою асимптотичною точністю. Дуже важливою особливістю цього методу є здатність робити параметр $\epsilon$ скільки завгодно малим. Це досягається вибором скільки завгодно тонкого примежового шару. Така форма розв'язку задовольняє крайовій умові, задача зводиться до отримання нетривіального розв'язку рівняння Лапласа. У даній статті був розроблений алгоритм застосування методу сингулярно- збурених рівнянь для задачі Діріхле у колі, а також на чисельних прикладах було доведено доцільність його застосування. Загалом даний метод може бути застосований для областей, обмежених кривими другого порядку, якщо вони є ліпшицевими.

\section{Бібліографічні посилання}

1. Вайнштейн Л. А. Открытые резонаторы и открытые волноводы / Л. А. Вайнштейн. - М. : 1966 - 475 с.

2. Михлин С. Г. Курс математической физики / С. Г. Михлин -М. : Наука, 1968.

3. Ostapenko $V$. A. Method of the singularly perturbed differential equations in internal problems of diffraction in polygonal domains in Proc. 11th International Conference on Mathematical Methods in Electrjmagnetic Theory (MMET*06) / V. A. Ostapenko. Kharkov, 2006.-532-534 pp.

4. Положий Г. Н Уравнения математической физики / Г. Н. Положий -М. : 1964. - C.69-106.

5. Тихонов А. Н., Самарский А. А. Уравнения математической физики. - М. : Наука, 1977. 